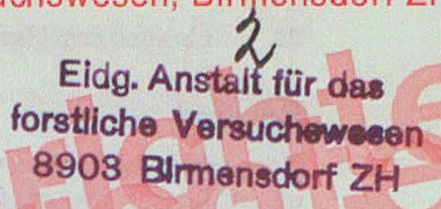

551.553 .12

Eidgenössische Anstalt für das forstliche Versuchswesen, Birmensdorf, Schweiz

\title{
Neuere Beobachtungen über die Entwicklung des Schönwetterwindsystems in einem V-förmigen Alpental (Dischmatal bei Davos)
}

\author{
Charlotte Urfer-Henneberger
}

Mit 9 Abbildungen

Eingegangen am 8. September 1969

\section{Zusammenfassung}

Durch neu hinzugewonnene Erkenntnisse und von verschiedenen Seiten beigetragene Beobachtungen konnte das erstmals 1964 publizierte Schema des tagesperiodischen Windsystems im Dischmatal in einigen Punkten verbessert und abgeändert werden. Als Erläuterung zu Abb. 5 wird es im einzelnen besprochen. Vergleichsweise werden auch Beobachtungen, Messungen und Überlegungen anderer Autoren aus der neueren Literatur herangezogen. Selten stehen diese im Widerspruch zu unseren Ergebnissen; meistens ergänzen und präzisieren sie diese. Elementare Faktoren, wie Strahlung, Hangexposition und Talrichtung, beeinflussen das lokale Windsystem in entscheidender Weise.

\section{Summary}

New Observations of Mountain and Valley Winds during Undisturbed Clear Days in a V-Shaped Alpine Valley (Dischma Valley near Davos, Switzerland)

Since publication of a first mountain and valley wind system in the Dischma valley in 1964, several new findings and observations were made which allow some improvements and modifications of the original scheme. As an explanation of Fig. 5, the new scheme is discussed in detail. Comparisons with new results published by other authors indicate few contradictions to our findings, but contribute various completions. It is emphazised that the valley wind system is decisively influenced by elementary factors like radiation, slope exposure, and direction of the valley.

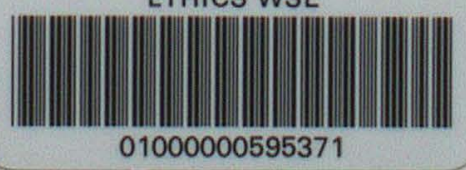




\section{Résumé}

Nouvelles observations des courants aériens par beau temps non perturbé dans une vallée alpine en forme de "V" (Vallée de Dischma près de Davos, Suisse)

Des résultats de mesures complémentaires et des rapports d'observations parvenus de divers côtés contribuent maintenant à l'amélioration de certains points du modèle de circulation des courants aériens dans la vallée de Dischma établi en 1964. Les 8 phases de la Fig. 5 sont discutées. A titre comparatif nos résultats sont commentés et discutés avec les travaux récents d'autres chercheurs. Pour la plupart ils complètent et précisent nos investigations antérieures. Des facteurs élémentaires comme le rayonnement, l'exposition de la pente et l'orientation de l'axe de la vallée jouent un rôle important dans la circulation locale des courants aériens de la vallée.

\section{Riassunto}

Nuove osservazioni sulle condizioni anemologiche nei giorni di bel tempo anticiclonico in una valle alpina a forma di "V" (Valle di Dischma presso Davos)

Grazie alle nuove conoscenze acquisite e alle osservazioni fornite da diverse parti è stato possibile migliorare e modificare in alcuni punti lo schema, pubblicato per la prima volta nel 1964, del sistema dei venti a periodo giornaliero nella valle della Dischma.

Quali spiegazioni alla figura 5 tale sistema viene trattato in dettaglio. Per il confronto vengono utilizzate anche osservazioni, misure e considerazioni di altri autori, presi dalla letteratura più recente. Raramente sono in contraddizioni con i nostri risultati; per lo più le completano e precisano. Fattori elementari, come radiazione solare, esposizione di pendio e direzione della valle, influenzano il sistema dei venti locali in modo decisivo.

\section{Problemstellung und Versuchsanordnung ${ }^{1}$}

Im Rahmen eingehender forstlicher Standortserkundungen wurden im Jahre 1959 von der Eidgenössischen Anstalt für das forstliche Versuchswesen (EAFV) im Dischmatal bei Davos mehrere meteorologische Stationen eingerichtet und mehrheitlich bis 1966 in Betrieb gehalten, allerdings jeweils nur in der schneefreien Vegetationsperiode. Man verfolgte dabei das Ziel, eine am linken Talhang in einer Höhe von rund $2000-2250 \mathrm{~m}$ ausgewählte Versuchsfläche für Hochlagenaufforstung (speziell im subalpinen Lawinenanrißgebiet) in das Klima eines größeren Talbereiches einzuordnen.

Auf dieser Versuchsfläche (auf der Stillbergalp) wird vornehmlich der Einfluß der Umweltfaktoren (Mikroklimate, Vegetation, Boden u.a.) auf das Wachstum von Aufforstungen studiert [25], mit der besonderen Absicht, auf einem Teil des steilen und stark gegliederten Versuchsgeländes auch die Wechselwirkungen zwischen Lawinenanbrü-

1 Von H. TUrner. 


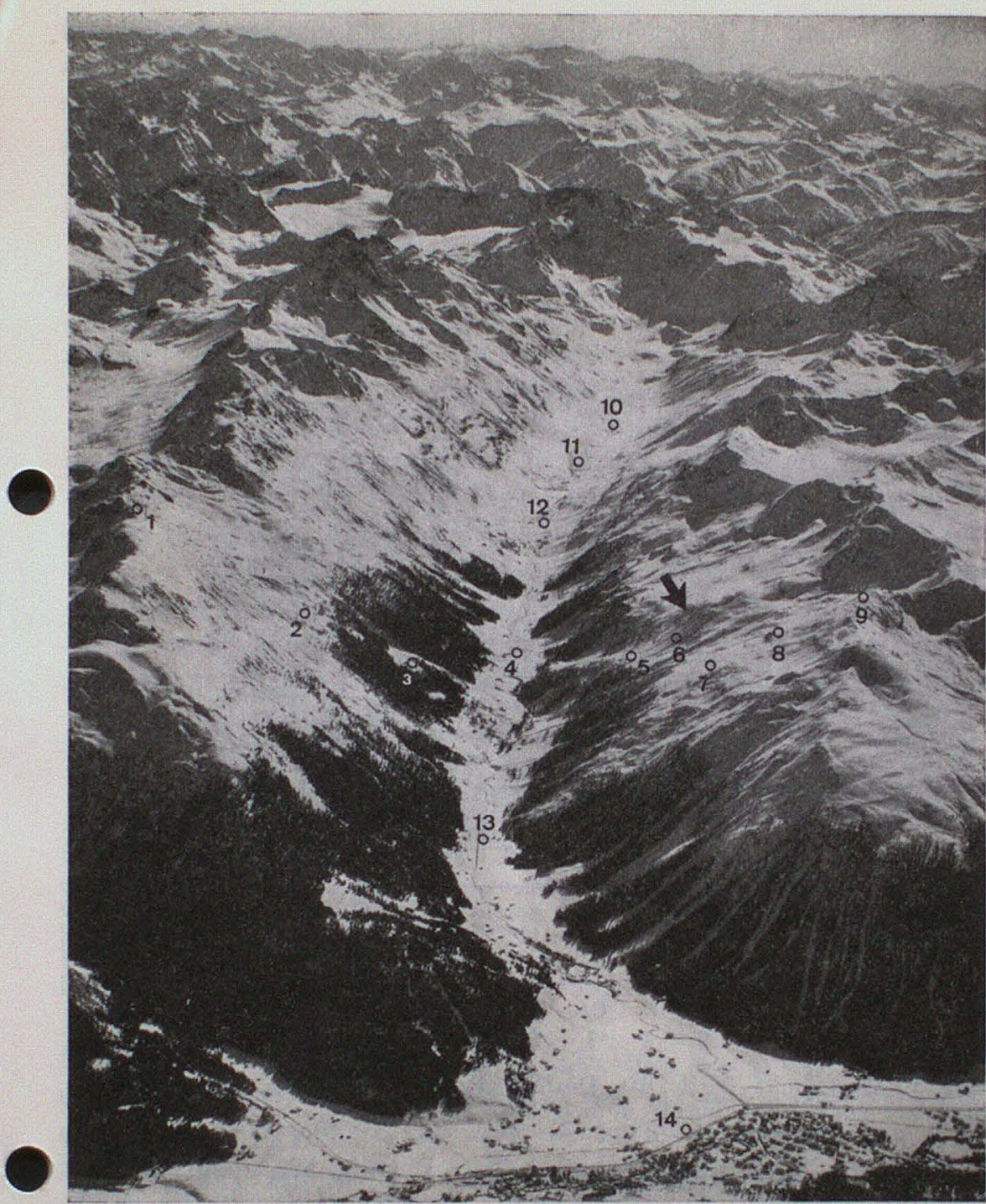

Abb. 1. Flugaufnahme des Dischmatales bei Davos, Ansicht gegen Südosten, mit den meteorologischen Stationen:
1 Baslerkopf $2536 \mathrm{~m}$
2 Lucksalp $2104 \mathrm{~m}$
6 Versuchsfläche $2094 \mathrm{~m} 11$ Jenatschenalp $1920 \mathrm{~m}$
3 Chalet Jäger $1870 \mathrm{~m}$
7 Stillberg $2130 \mathrm{~m}$
12 Gadmen $1820 \mathrm{~m}$
8 Gyrenspitz $2364 \mathrm{~m}$
13 Kaisern $1590 \mathrm{~m}$
4 Teufi $1700 \mathrm{~m}$
9 Brämabüel $2560 \mathrm{~m}$
14 Davos $1550 \mathrm{~m}$
5 Ob Mäder $1960 \mathrm{~m}$
10 Dürrboden $2000 \mathrm{~m}$

Der Pfeil weist auf das im Bereich der Stillbergalp gelegene Versuchsgelände, wo mikroklimatisch-ökologische Forschungen zum Projekt „Aufforstung im Lawinenanrißgebiet" durchgeführt werden.

Photo und Bewilligung zur Reproduktion durch den Militärflugdienst Dübendorf 
chen und dem Aufkommen einer Aufforstung zu untersuchen. Das Eidgenössische Institut für Schnee- und Lawinenforschung Weißfluhjoch (SLF) ist an diesen Untersuchungen maßgeblich beteiligt.

Das Dischmatal beginnt in der vergletscherten Grialetsch-ScalettaGebirgsgruppe und mündet nach einem $15 \mathrm{~km}$ langen, fast geradlinig SSE - NNW gerichteten Verlauf bei Davos in das Landwassertal (Abb. 1). Es hat - mit einer Sohlenhöhe von etwa 1600-2000 m den Charakter eines Hochtales. Die beidseitigen Kammlinien erheben sich ziemlich einheitlich $900-1000 \mathrm{~m}$ über die Talsohle. Im Talschluß sind links und rechts des vergletscherten Piz Grialetsch $(3131 \mathrm{~m})$ als Übergänge ins Engadin zwei verhältnismäßig hohe Pässe eingesenkt (Scalettapaß, $2606 \mathrm{~m}$, und Fuorcla da Grialetsch, $2537 \mathrm{~m}$ ). Die vordere Hälfte des Tales ist bis in eine Höhe von etwa $2000 \mathrm{~m}$ mit stark verlichtetem, lawinendurchzogenen Lärchen-Fichtenwald und Lärchen-Zirbenwald bestockt, während der hintere Teil völlig waldlos ist.

Wie das Ubersichtsbild (Abb. 1) erkennen läßt, erstrecken sich die mesoklimatischen Beobachtungsstationen (1-9) über einen Querschnitt im vorderen Drittel des Tales. Dieses Profil, dargestellt in

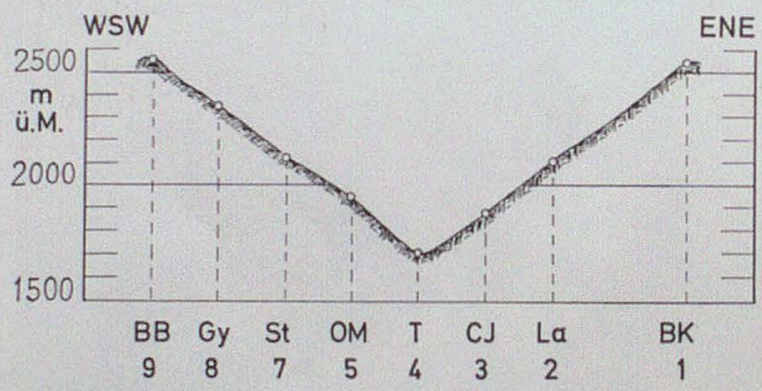

Abb. 2. Querschnitt durch das Dischmatal im Bereich der meteorologischen Stationen $1-9$ (Normalprofil, nicht erhöht)

Abb. 2, offenbart eine ausgesprochene V-Form des Tales. Außer Windweg und Windrichtung wurde an diesen Stationen auch Temperatur, Feuchtigkeit und Niederschlag registriert (vgl. Abb. 3). Die hier eingesetzten Windweg- und Windrichtungsschreiber sind Konstruktionen von P. Rochat (EAFV) und wurden von W. NäGELI [19] genau beschrieben. Ein Kontaktanemometer mit einem sehr leichten, sechsteiligen Schalenstern in $1,7 \mathrm{~m}$ über Boden registriert den Windweg, indem jede hundertste Umdrehung des Schalensterns auf einem ablaufenden Schreibband mit $60 \mathrm{~mm}$ Vorschub pro Stunde markiert wird. Die Anlaufschwelle des Systems liegt bei $0,1 \mathrm{~m} / \mathrm{sec}$. 
Die Windrichtung wird wirkungsvoll durch eine leichte Windfahne mit sehr langem Arm gedämpft und auf dem gleichen Schreibband durch etwa 80 Richtungspunkte pro Stunde registriert.

Die beiden Kammstationen Brämabüel $(2560 \mathrm{~m})$ und Baslerkopf $(2536 \mathrm{~m})$, die beiden Hangstationen Stillberg $(2130 \mathrm{~m})$ und Lucksalp $(2104 \mathrm{~m})$ sowie die Talstation Teufi $(1700 \mathrm{~m})$ standen in der schnee-

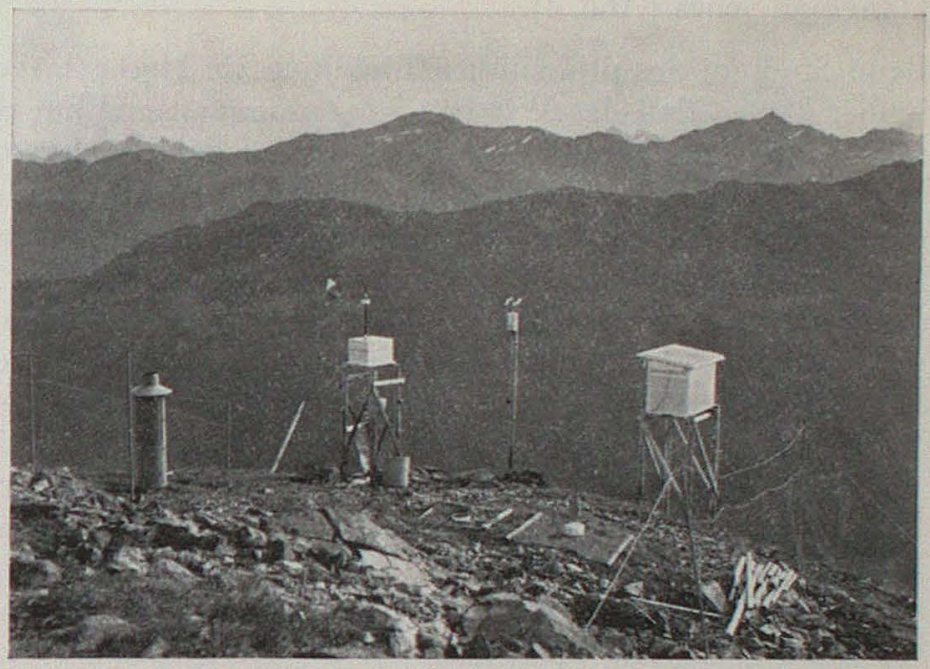

Abb. 3. Meteorologische Station Brämabüel, $2560 \mathrm{~m}$, auf dem linksseitigen Kamm des Dischmatales. Ansicht gegen Nordosten. Von links nach rechts: Regenschreiber nach Hellmann, Windfahne und Windweg-Windrichtungsschreiber Modell Rochat, Kontaktgeber für Windweg Modell Rochat, davor zwei in den Boden eingesenkte Hellmann-Regenmesser (einer mit Nebelfänger nach Grunow), englische Wetterhütte Photo: W. NÄGELI (Oktober 1961)

freien Zeit der Jahre 1959 bis 1961 in Betrieb. Zur genaueren Erfassung der klimatischen Verhältnisse an den unteren Hangteilen wurden in den Folgejahren bis 1966 die beiden Kammstationen aufgelassen und dafür auf der gleichen Profillinie an vier weiteren Stellen (Gyrenspitz, 2364 m, Versuchsfläche Stillbergalp, 2094 m, und Ob Mäder, $1960 \mathrm{~m}$, auf der linken Talseite, Chalet Jäger, $1870 \mathrm{~m}$, auf der rechten Talseite) Stationen errichtet. Im Jahre 1961 wurden die Untersuchungen ergänzt durch Wind- und Temperaturbeobachtungen an fünf weiteren Stationen (Nr. 10-14 in Abb. 1) in der Tallängsachse.

Das in vorliegender Arbeit behandelte Schönwetterwindsystem ist für die Kenntnis des gesamten Windregimes in diesem Alpental von großer Bedeutung, denn es läßt sich zeigen, daß der sogenannte 
Schönwetterwind keineswegs auf störungsfreies Hochdruckwetter beschränkt ist. Vielmehr setzt sich das tagesperiodische Zirkulationssystem der Tal-, Berg- und Hangwinde auch in allen anderen Wetterlagen mehr oder weniger durch, so daß die durchschnittlichen Windverhältnisse, zumindest während der schneefreien Zeit, sehr stark an jene bei Schönwetter angenähert sind; ja selbst die Windverteilung in den Niederschlagsperioden zeigt noch deutliche Anklänge an jenes (Abb. 4).

Beim Studium des pflanzlichen Bioklimas im Hochgebirge zeigt sich immer klarer, daß der Wind - im Zusammenspiel mit dem Relief, mit der Strahlung und mit der Schneedecke - die pflanzlichen Wuchs- und Lebensbedingungen so stark beeinflußt, daß ihm in diesem Bereich der Charakter eines ausgesprochenen Entscheidungsfaktors zukommt. Im Hochgebirge spielt bekanntlich der Austausch fühlbarer Wärme zwischen der Boden- und Pflanzenoberfläche und der Atmosphäre im allgemeinen eine wesentlich größere Rolle als im Tiefland, während der Energieverbrauch für Verdunstung eher zurücktritt und die Exposition des Geländes zu Wind und Strahlung diese Energieströme und damit den gesamten Wärme- und Wasserhaushalt außerordentlich stark variiert. Darin liegt die wohl größte Bedeutung genauer Kenntnisse über das bodennahe Windfeld für den im Gebirge mit Kulturaufgaben betrauten Wirtschafter. Daneben ist es für ihn wichtig, zu wissen, in welchen Zeiten und Räumen der Wind, beispielsweise durch Verbreitung der Sporen pathogener Pilze, den Erfolg von Kulturen in Frage stellen kann. (Pilzkrankheiten spielen in vielen subalpinen Aufforstungen eine verheerende Rolle.) Das Windsystem im Berggelände ist ferner für die Samenverbreitung der meisten Baumarten von unmittelbarer Bedeutung. Auch die Tatsache, daß bei der Pflanzennachzucht von Baumarten-Ókotypen aus den Hochlagen immer wieder aberrante Vertreter vorkommen, die physiologisch und morphologisch den Tiefland-Rassen gleichen, ist am ehesten durch die Pollenverbreitung aus tieferen in höhere Lagen mit den Tal- und Hangaufwinden erklärbar.

Erste Ergebnisse über die Wind- und Temperaturverhältnisse an ungestörten Schönwettertagen im Dischmatal wurden von Frau Dr. UrFer-HenNEBERGER im Jahre 1964 vorgelegt; ein vorläufiges Schema des Hang- und Talwindsystems war darin bereits enthalten ([28], Fig. 13, S. 414). In Fortsetzung ihrer Auswertung des Registriermaterials konnte sie an anderer Stelle [29] mit Hilfe der potentiellen Äquivalenttemperatur, bzw. ihrer Differenz zwischen den einzelnen Meßstellen, die Gesetzmäßigkeiten der verschiedenen Strö- 
mungsphasen noch deutlicher abgrenzen; namentlich ergaben sich weitere Hinweise dafür, daß abends ein Querwind vom Osthang
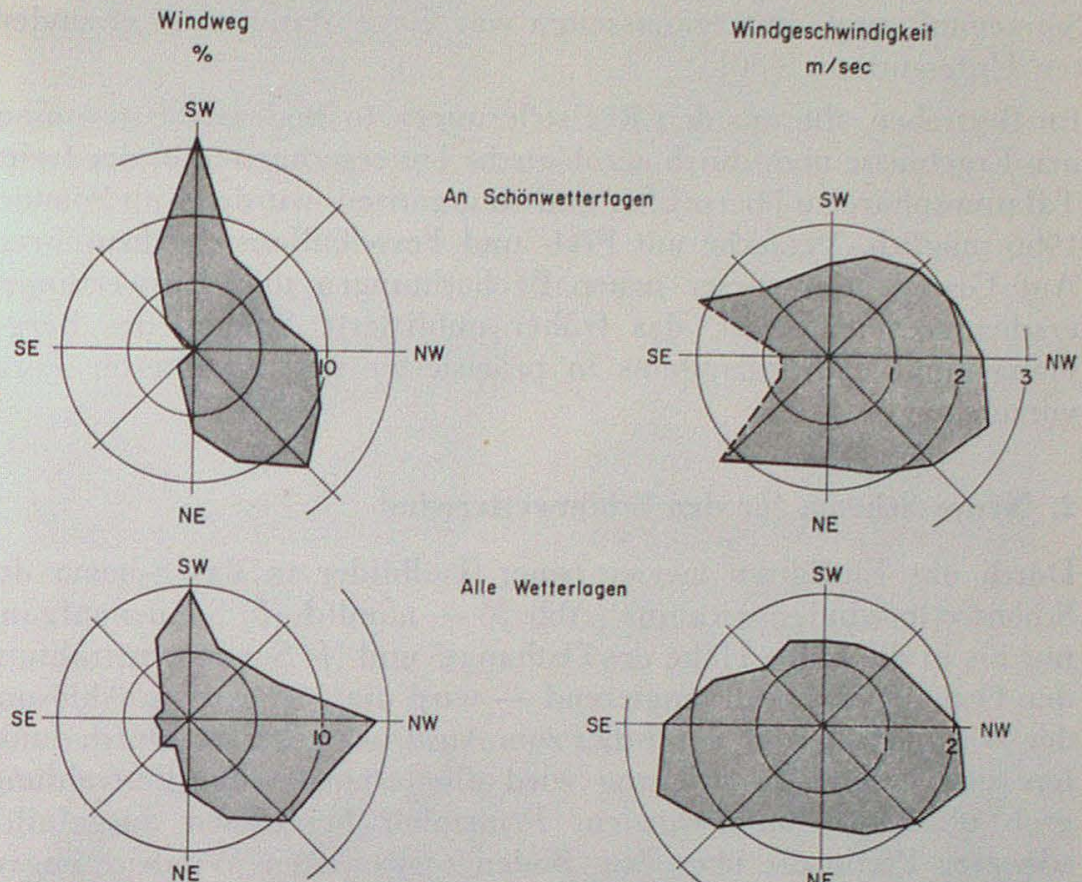

Alle Wetterlogen
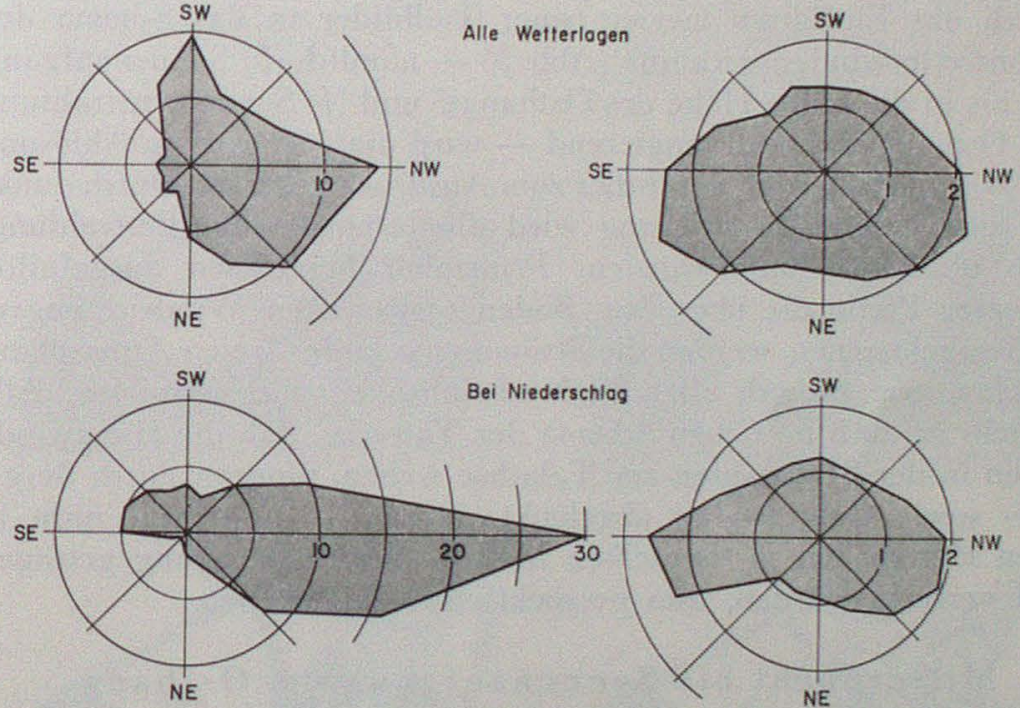

Abb. 4. Windrosen der meteorologischen Station Versuchsfläche Stillbergalp, $2094 \mathrm{~m}$, am linksseitigen Hang des Dischmatales (nach H. Turner [26])

a) für 42 ungestörte Schönwettertage (Juli-September 1963-1965); b) für die Monate Juni-September 1962-1965; c) für Niederschlagsperioden (722 Stunden, Juni-September 1962-1965)

Von den Komponenten des Schönwetterwindsystems des ganzen Tales fehlt an dieser Hangstation, rund $300 \mathrm{~m}$ über dem Talboden, lediglich der nächtliche Talauswind. Bei örtlichem Sonnenaufgang Windsprung von SW (Hangabwind) nach NE (Hangaufwind), hernach stetige Linksdrehung des Windes. Morgens bis mittags: Hangaufwind, drehend von NE nach N. Nachmittags: Taleinwind NNW-NW. Abends (etwa 19-21 Uhr): Westwind schräg hangabwärts/taleinwärts. Nachts: Hangabwind SW (vgl. Abb. 5) 
über den Talabwind hinweg zum Westhang weht und daß nachts die Hangabwinde den Talgrund nicht erreichen. Die schon früher festgestellte auffällige Gebundenheit der Windphasenwechsel an die Sonnenauf- und -untergangszeiten war Gegenstand einer gesonderten Untersuchung [30].

Im Bestreben, die aus den Registrierungen in Bodennähe gewonnenen Ergebnisse noch durch aerologische Untersuchungen in der freien Talatmosphäre zu überprüfen und zu ergänzen, wurde es im Sommer 1966 möglich, Versuche mit Frei- und Fesselballons durchzuführen. Auf Grund aller dieser neuen Beobachtungen und Auswertungen erscheint es angezeigt, das früher publizierte Schema des Berg-, Hang- und Talwindsystems in präzisierter und erweiterter Form vorzulegen.

\section{Neues Schema für den Schönwetterwind}

Durch das Einführen zweier neuer Teilbilder in das Schema der Schönwetterwindentwicklung (Abb. 5) - nämlich $(b)$ Sonnenaufgang nur bis in die halbe Höhe des Osthangs ${ }^{2}$ und $(f)$ Sonneneinstrahlung den Osthang nur noch tangierend - wird die gegenseitige Ablösung der Windphasen noch deutlicher zum Ausdruck gebracht. Durch dunklere und hellere Schattierung wird die Intensität der Bestrahlung, grob abgestuft, unterschieden. Prinzipiell bezeichnen ausgefüllte, schwarze Pfeile die über dem Boden registrierten Windrichtungen, die leergelassenen, weißen die Strömungen in der freien Atmosphäre darüber, wie sie sich aller Wahrscheinlichkeit nach abspielen. Allgemein ist auch im neuen Schema der Tatsache, daß die Hangwinde selten in der Senkrechten zur Talachse wehen, sondern durch Berg-, Tal- sowie Gebirgswind abgelenkt werden - und zwar nicht in allen Hanghöhen in demselben Maß - besser Rechnung getragen und versucht worden, dies perspektivisch darzustellen.

\subsection{Mitternacht bis Sonnenaufgang am Osthang (Abb. $5 \mathrm{a})$}

Der Bergwind fließt in den frühen Morgenstunden genau in der Talachse gleichmäßig laminar talabwärts und hat eine Mächtigkeit in der Größenordnung von ein paar hundert Metern (Pilotballon-

2 Zur Vermeidung von Mißverständnissen sei darauf hingewiesen, daß in der vorliegenden Arbeit die Seitenhänge des Tales (entsprechend der von R. Geiger [12, S. 388] aufgestellten Definition) nach ihrer Exposition zur Besonnung bezeichnet sind; so bedeutet z. B. "Osthang" den das Tal auf der westlichen Seite begrenzenden Talhang. Die Red. 


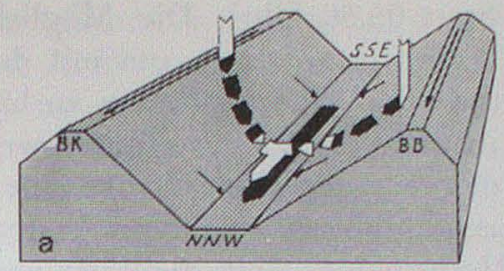

Mitternacht bis Sonnenaufgang am E-Hang

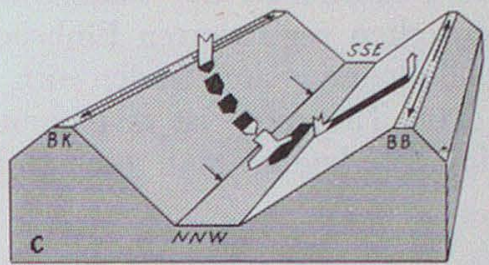

Ganzer E-Hang besonnt, auch im Tal wind die Sonne aufgehen

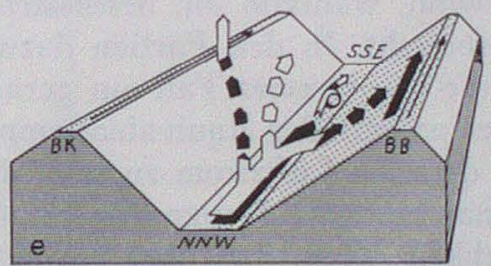

Westhang stärken besonnt als E-Hang

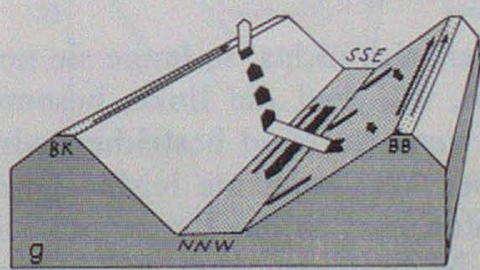

Sonnenuntengang am E-Hang und im Talgrund

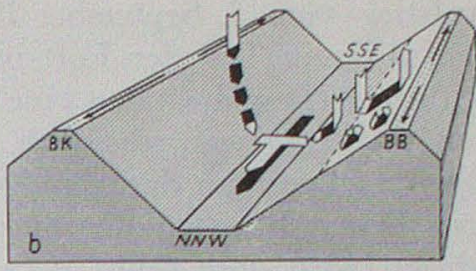

Sonnenauigang am oberen E-Hang

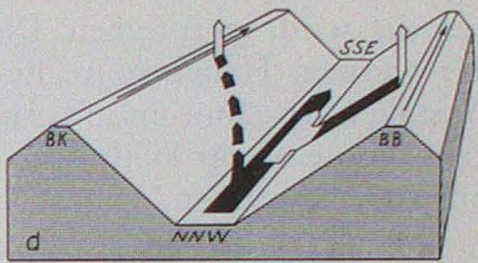

Ganzes Tal besonnt

Einsatzzeit des Talwindes in der ganzen Länge des Tales zur selben Zeir

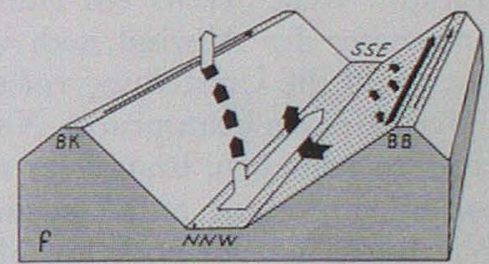

Sonneneinstrahlung am E-Hang nur noch tangierend

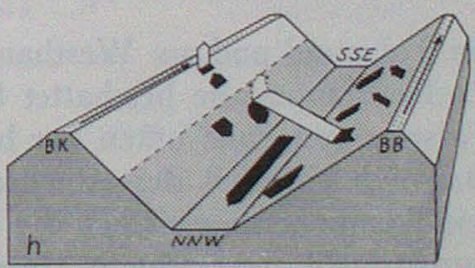

Nach Sonnenuntergang im Tal und am unteren Westhang

Abb. 5. Verbessertes Schema der Strömungslinien in den Grundschichten eines V-förmigen SSE-NNW-Tales. Länge und Breite der Pfeile haben - mit Ausnahme der „einzelnen Luftpakete, die nachts einfallen“ - nichts zu tun mit der Geschwindigkeit oder Mächtigkeit der betreffenden Windphasen; sie wurden aus zeichnerischen Gründen mehr oder weniger breit und lang gezeichnet. Bezüglich Windweg und Geschwindigkeit der einzelnen Strömungsphasen am Hang vgl. Abb. 4 
Aufstieg vom 7. September 1966, Start 05.56 Uhr). Die Möglichkeit, daß ein Teil der Luft in der Talmitte aufsteigt, um mit den seitlichen Hangabwinden eine geschlossene Windzirkulation zu bilden, ist wenig wahrscheinlich. Das in der Literatur erwähnte zweigeteilte Maximum der Windverteilung mit der Höhe dürfte einerseits dem Abfließen der durch Ausstrahlung abgekühlten Bodenschicht (wird doch selbst noch im Hochsommer dort die Luft nach der potentiellen Äquivalenttemperatur einige Grade kälter als die übrige Luft innerhalb des Tales, Abb. 6) und andererseits dem eigentlichen Bergwind, der aus dem Nachfließen der Hangwinde gespeist wird, entsprechen. Die vereinzelten registrierten Einbrüche der warmen Hangluft in die kalte Bodeninversion finden sich in Aussagen der Anwohner bestätigt. („Im Tal unten ist es frühmorgens meistens sehr kühl; aber für Momente kommt man ganz plötzlich zum Erwarmen.")

\subsection{Sonnenaufgang am oberen Osthang (Abb. 5 b)}

Charakteristisch sind die ersten Anderungen, wenn der Osthang etwa bis zur Mitte in der Sonne steht: Im oberen, besonnten Hangabschnitt weht bereits ein Hangaufwind, während am beschatteten Unterhang der Abwind noch weiterbesteht. In den Partien dazwischen geht die Umstellung vor sich, die im extremen Fall nur gerade einige Minuten beansprucht. Nach der potentiellen Äquivalenttemperatur zu schließen, braucht es nicht derselbe Luftstrom zu sein, der von allem Anfang an schon die ganze besonnte Hangpartie erfaßt. Wir lassen darum noch die Möglichkeit offen, daß über erhitzten Zonen auch nur lokale Verwirbelungen auftreten könnten.

\subsection{Ganzer Osthang besonnt (Abb. 5c)}

Im Talgrund und am Westhang ändert sich nichts, so lange sie noch in der Hauptsache beschattet bleiben, während die linke, besonnte Talseite von ganz unten her bereits vom Aufwind bestrichen wird. Daß sich ein Teil des Abwindes vom Westhang als leichte Querwindkomponente zwischen die untersten Partien des Bergwinds hindurchschiebt, beobachtete Herr Dr. M. SchüEPP. Ihre Mächtigkeit beträgt nur ein paar Dekameter, und sie berührt den Boden des V-Tales selber nicht; denn in den Registrierungen an der Station Talboden (Teufi) finden sich keine Andeutungen für einen morgendlichen Querwind. Dieser müßte theoretisch in den oberen Talpartien - vom beschatteten West- zum bereits besonnten oberen Osthang - beginnen und sich allmählich in die Tiefe durchsetzen, 
was unseren Vorstellungen zuwiderläuft. Hingegen ist anzunehmen, daß kurzfristig im nachlassenden Bergwind in einzelnen Talabschnitten eine Strömung auftritt, die von Ost nach West quer zum Tal gerichtet ist. Buettner und Thyer ([4], S. 142) haben tatsächlich beobachtet, daß frühmorgens ein ausgewichteter Pilotballon zuerst dem Abwind am Schattenhang folgte und dann vom Hangaufwind der anderen, bereits besonnten Talseite erfaßt und bis in die Höhe des gegenüberliegenden Kammes emporgetragen wurde.

\subsection{Ganzes Tal besonnt (Abb. 5d)}

In seiner ganzen Länge mehr oder weniger gleichzeitig setzt im Talgrund der Talwind ein und erfaßt die untersten und sehr bald auch schon als Hangaufwind die oberen rechten, schattseitigen Hangpartien. Da die potentielle Äquivalenttemperatur vom Talboden und Westhang unter sich gleich verlaufen, zu denen am Osthang dagegen noch über Stunden eine große Differenz besteht (Abb. 6), schließen

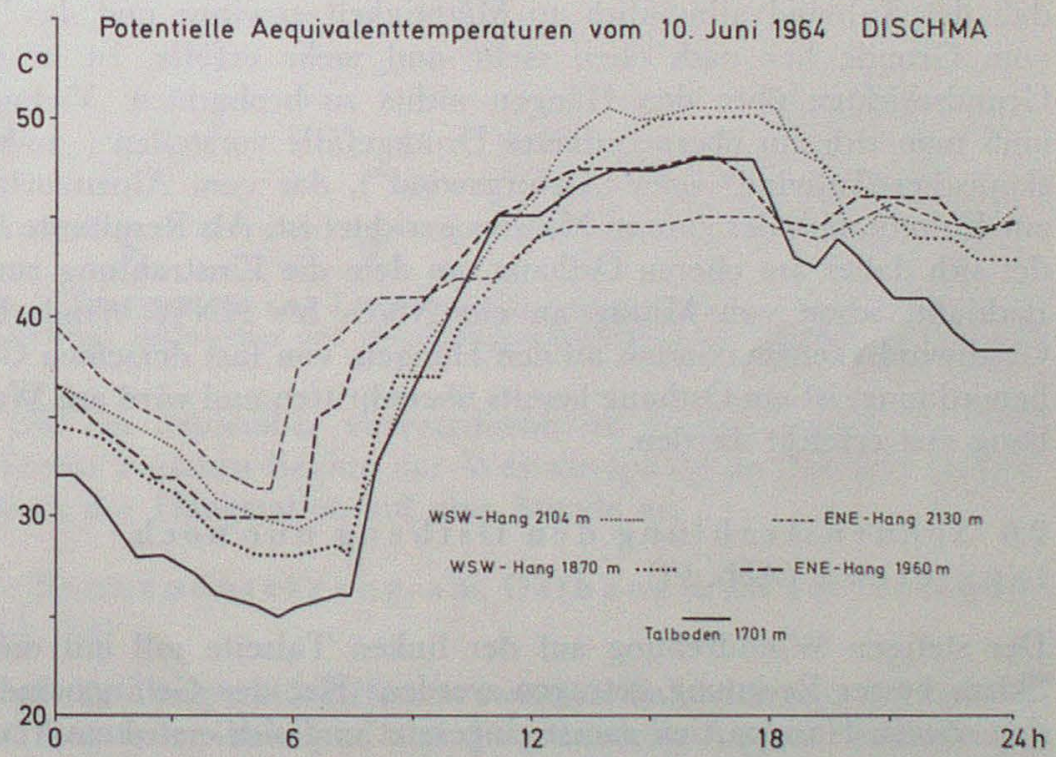

Abb. 6. Tagesgang der potentiellen Äquivalenttemperatur an ungestörtem Schönwettertag (10. Juni 1964)

wir, daß die eine Luftmasse dem Talboden und den nach Westen orientierten, noch schattigen Hängen nach hinaufstreicht, während der Nachschub am besonnten Gegenhang aus der freien Atmosphäre im Talinnern kommen muß. 
2.5. Westhang stärker besonnt als Osthang (Abb. 5 e) Bald erfüllt eine einheitliche Luftzufuhr das ganze Tal. Die potentiellen Äquivalenttemperaturen der beiden Seiten haben sich angeglichen. Im Gegensatz zum laminaren Bergwind ist der Talwind sehr turbulent und weht nicht genau in der Talrichtung, sondern pendelt ständig; seine Richtung umfaßt einen breiten Fächer gegen die Hänge hin; man möchte ihm stellenweise auch eine "Korkzieher"Bewegung zuschreiben (die Pilotballone erfahren sowohl Auf-als auch Abwinde auf ihrer Flugbahn).

Entsprechend der nachlassenden bzw. zunehmenden Einstrahlung am Ost- und Westhang unterliegt bzw. überwiegt die Komponente des Hangaufwinds gegenüber derjenigen des Gebirgswinds. Die Drehung vom Hangaufwind in eine mehr talparallele Richtung ist zuerst an den oberen und erst Stunden später an den unteren Meßstellen zu beobachten. In $260 \mathrm{~m}$ Höhe über dem Talboden dreht die Windfahne erst zu Anfang des Nachmittags auf N. Von der Theorie, daß der Talwind allmählich an Mächtigkeit gewinne und das Tal vom Grunde her nach oben mehr und mehr erfülle, ist in den Grundschichten über den Hängen nichts zu beobachten. Vielmehr muß man sich ein übergeordnetes Druckgefälle vorstellen ("makroskopischer Talwind" oder "Gebirgswind“), das vom Alpenvorland zur Kulmination des ganzen Massivs gerichtet ist. Als Resultante findet sich daher am oberen Osthang, an dem die Einstrahlung zuerst nachläßt, schon von Mittag an ein NNE- bis NNW-Wind. Das Geschwindigkeitsmaximum an den Hängen, von fast derselben Größenordnung, ist am Osthang bereits überschritten und wird am Westhang erst erreicht werden.

\subsection{Sonnenstrahlung den Osthang nur noch tangierend (Abb. $5 \mathrm{f}$ )}

Der stetigen Winddrehung auf der linken Talseite soll mit dieser Skizze besser Rechnung getragen werden: Hat der Gebirgswind in den oberen Hangpartien zuerst eingesetzt und sich erst danach auch in den mittleren allmählich durchgesetzt, so hält er hier nun auch länger an, während im unteren Osthang eine neue Umstellung vor sich geht. Schon vor örtlichem Sonnenuntergang tritt die Hangabwind-Komponente in Erscheinung, zuerst als W-Wind im Talgrund, als WNW in der Hangmitte und noch als NW weiter oben. Die Westwindphase im Tal, als Resultante zwischen Hangab(WSW) und Talaufwind (WNW), tritt ein (Abb. 7), wenn bei dem 
raschen Temperaturrückgang am untersten Talhang die Temperaturdifferenz zur Station am Talgrund die Trockenadiabate erreicht, und hält praktisch so lange an, bis sich die Talsohle gegenüber dem Hang unterkühlt. Die Auszählung der Einsatzzeiten ergab, daß sich die mittlere Einsatzzeit des Westwindes von 17 Uhr im Sommer auf

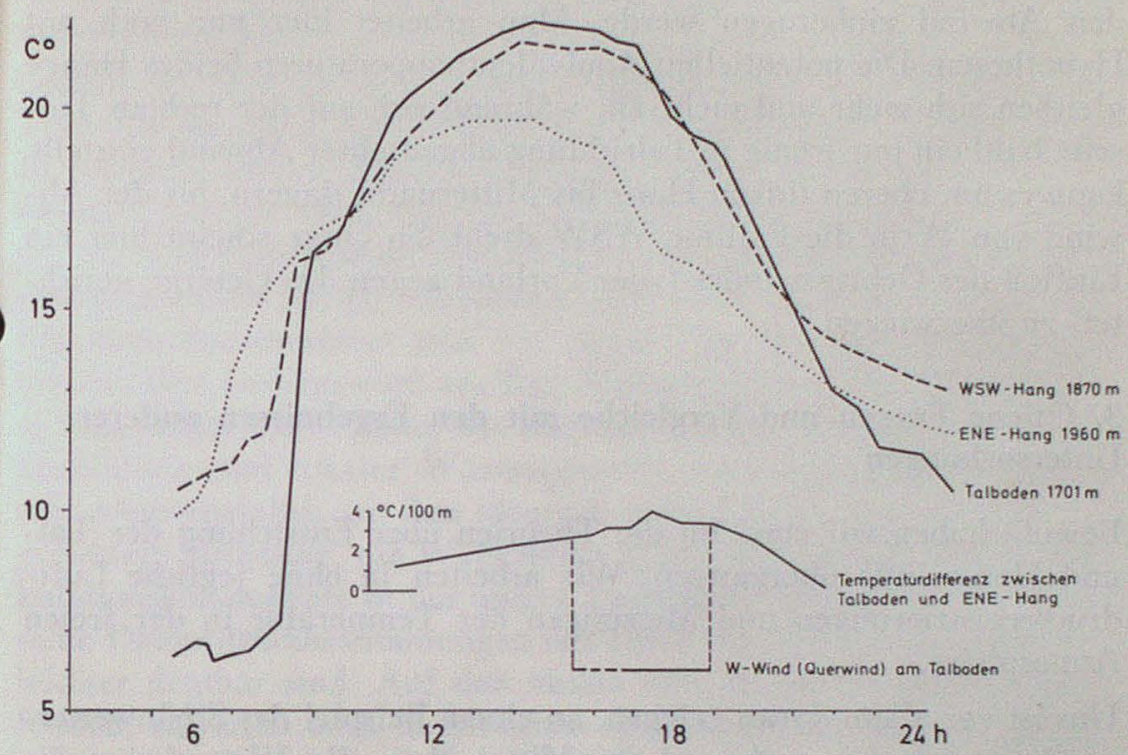

Abb. 7. Temperaturen und Temperaturdifferenzen in den untersten Talpartien bei ungestörtem Schönwetter und gleichzeitigem Querwind am Talboden (26. Juli 1964)

14 Uhr im September vorverschiebt; im Herbst wächst die Zeitdifferenz zwischen Beginn der Westwindphase im Tal und derjenigen an der Hangstation auf eine Stunde an.

2.7. Sonnenuntergang am Osthang und im Talgrund (Abb. $5 \mathrm{~g})$

Wenn die Temperaturdifferenz zwischen Talboden und $260 \mathrm{~m}$ höher am Hang unter drei Grad und die potentielle Äquivalenttemperatur im Tal unter diejenige der übrigen Stationen absinkt, geht im Talgrund die Westwindphase zu Ende und besteht vermutlich nur noch in den Luftschichten darüber. An der Talsohle kann sich manchmal noch für kurze Zeit der Talwind einstellen; meistens geht aber die Umstellung auf den Bergwind vor sich. An den untersten nach Osten orientierten Hängen weht nun der Abwind in der Fallinie, während darüber die Drehung stetig vor sich geht. 
2.8. Nach Sonnenuntergang im Tal und am unteren Westhang (Abb. $5 \mathrm{~h})$

Da schon vor örtlichem Sonnenuntergang am Westhang der Aufwind zusammenbricht und bald der Abwind in den unteren Partien einsetzt, muß man sich fragen, ob der Querwind dementsprechend in den Abwind einbezogen werde. Man arbeitet hier nur noch mit Hypothesen: Die potentiellen Äquivalenttemperaturen beider Hänge gleichen sich mehr und mehr an; während sich auf der rechten Talseite bald ein nur wenig in Talrichtung abgedrehter Abwind einstellt, kann es am oberen linken Hang bis Mitternacht dauern, bis der Abwind von W in die Fallinie WSW dreht. So lange scheint hier ein Einfluß des Gebirgswindes (vom Vorland gegen das Gebirge gerichtet) $\mathrm{zu}$ überwiegen.

\section{Offene Fragen und Vergleiche mit den Ergebnissen anderer Untersuchungen}

Bewußt haben wir zunächst die Theorien über Entstehung der Talund Hangwinde übergangen. Wir arbeiten ja ohne jegliche Luftdruckregistrierungen und Messungen der Temperatur in der freien Atmosphäre.

Uns ist vor allem daran gelegen, an einem Beispiel des Schönwetterwindes - der ja auch noch im Mittel über alle Wetterlagen die Dominanz in der Windrichtung besitzt (Mäder [17], EKHART [10]) und in komplexen Lagen den Gradientwind überlagert oder ablenkt - alle uns zur Kenntnis gekommenen Einzelheiten zu beschreiben. Da es sich im Dischma um ein einheitliches SSE-NNW-Tal handelt, lassen sich gewisse Gesetzmäßigkeiten oder mindestens das Prinzip ihrer Entstehung auf ähnlich exponiertes Gelände übertragen. Mit Buettner und Thyer [3], Jelinek [14], Berg [2], W. Schüepp [20], MacHattie [16] und anderen möchten wir die Betonung darauf legen, daß die Orographie wegen der Besonnungsverhältnisse jeden Ortes ("orographic winds, vents orographiques") bestimmt, in welcher Art und welchem Ausmaß bei Wegfall der Gradientwinde die lokalen Hang-, regionalen Tal-, großräumigen Gebirgs- und eventuellen Konvektionswinde dominieren oder einander überlagern.

Eine nicht weit zurückliegende und klare Übersicht über die Talund Hangwind-Theorien finden wir bei STERTEN [22] und zuvor bei Sterten und Knudsen [23]. Die früher datierte Arbeit enthält Wetterkarten mit Isobaren und die spätere Messungen des Luft- 
drucks längs eines Tales sowie des Windes in der freien Atmosphäre. Aus dem Gebiet der Schweizer Alpen ist eine Arbeit von KuHN [15] zu erwähnen: Beim Beibringen technischer Daten als Unterlagen für einen Alpentunnelbau stößt er auf ein mittleres Luftdruckgefälle tagsüber (13.30 Uhr) vom Alpenvorland gegen das Innere des Gebirges und für die Nacht (21.30 Uhr) auf eines im umgekehrten Sinn.

Gerade der Begriff des "Gebirgswindes" hilft, in die umstrittenen Theorien des Berg- und Talwindes mehr Ordnung und Systematik zu bringen. Diesen Begriff (Gebirgswind, ,vent du massif“) verwenden wir für eine über den Lokalwinden gelegene Strömung, die am Tag vom Vorland zum Gebirge und in der Nacht entgegengesetzt gerichtet ist. Der Phasenwechsel dürfte nur um wenige Stunden dem Sonnenunter- und vor allem dem Sonnenaufgang nachhinken. Den Gebirgswind am Tag (der eigentlich als "Vorlandwind“ zu bezeichnen wäre) sehen wir auch als Urheber ausgedehnter Wolkenbildung und lokaler Wärmegewitter am Alpenrand. Ferner ist sehr wahrscheinlich mit ihm identisch jene tagsüber gegen die Alpen gerichtete Strömung, die W. SchüEPP ([20], Abb. auf S. 39) als „Zirkulationskomponente in der untern Luftschicht" bezeichnet, durch die seine Dampfdruckbestimmungen mit Hilfe von Strahlungsmessungen leichter deutbar sind. Auf den nachts weit in das Vorland ausgreifenden, zuweilen noch in München spürbaren Gebirgswind, gleichgerichtet wie die bodennahen Kaltluftströme oder mit ihnen kombiniert, weist BAUMGartner [1] hin.

Tal- und Hangwindsysteme sind also noch von einer großräumigeren Zirkulation überlagert - von der WENGER als von einem "makroskopischen", Troller als von einem "übergeordneten Talwind" (beide zitiert bei EкHART [7]) sprechen -, auf die nun die ursprüngliche Streitfrage übertragen werden kann, ob es sich um hin- und herpendelnde Luft (HANN-SAIGEY) oder um eine geschlossene Zirkulation (Wenger-Fournet) handle. Ob so oder so, braucht der „Rückstrom" nicht mehr so eng gefaßt zu werden. Entsprechend der Volumvergrößerung umfaßt er noch ein Drittel oder Viertel der zuströmenden Luftmasse und deren Geschwindigkeit (WAGNER [32]); seine Richtung ist um etwa 180 Grad gegen den unteren Strom abgedreht, wodurch seine praktische Bedeutung, sicher im Einzelfall (BURGER und Eкhart [5]), dahinfällt. Der Rückstrom des Gebirgswindes kommt auch so hoch zu liegen ( $\geqq 4000 \mathrm{~m}$ in den Alpen), daß er nur in den seltensten Fällen nicht in einen Gradientwind einbezogen würde. In anderen Zonen (z. B. in Norwegen) oder in einheitlicherem 
Gelände (z. B. auf der Kuppe des Mt. Rainier) liegt das „überlagerte Windsystem" sehr viel tiefer und ist nicht mehr wesentlich verschieden vom eigentlichen "Antiberg- und -talwind“ (Buettner und Thyer [4]). Aber Ablenkungen und Verwirbelungen erfährt es auch dort noch durch überragende Kämme, benachbarte Talwindsysteme, Einflüsse möglicher Kontinentalwinde (Monsune) und Übergänge zum Wind der höheren Atmosphärenschichten, so daß man eigentlich sagen darf: Es ist keine Streitfrage, sondern Sache der Definition (STERTEN [22]), wie man das „Niemandsland“ zwischen den Talwinden und den Gradientwinden bezeichnen will.

Eine andere strittige Frage möchten wir wie folgt ergänzen: F. Defant [6] entwickelte eine Theorie der Hang-, Berg- und Talwinde, wobei er als treibende Kräfte voraussetzt, daß bei Hangaufwinden ein stark überadiabatisches Temperaturgefälle, bei Hangabwinden dagegen eine Bodeninversion oder Isothermie vorhanden sein müssen. Vergleicht man zwei Stationen am Berghang im Dischma miteinander, so mißt man bei Aufwind einen Gradienten von $0,5-1,0^{0}$ am Ost- bzw. $0,5-1,5^{0}$ am Westhang, während der Wert bei Hangabwinden in der Nacht auf beiden Talseiten $0,25-0,5^{0}$ beträgt (Abb. 8). Stellt man jedoch den Stationen in der Hangmitte

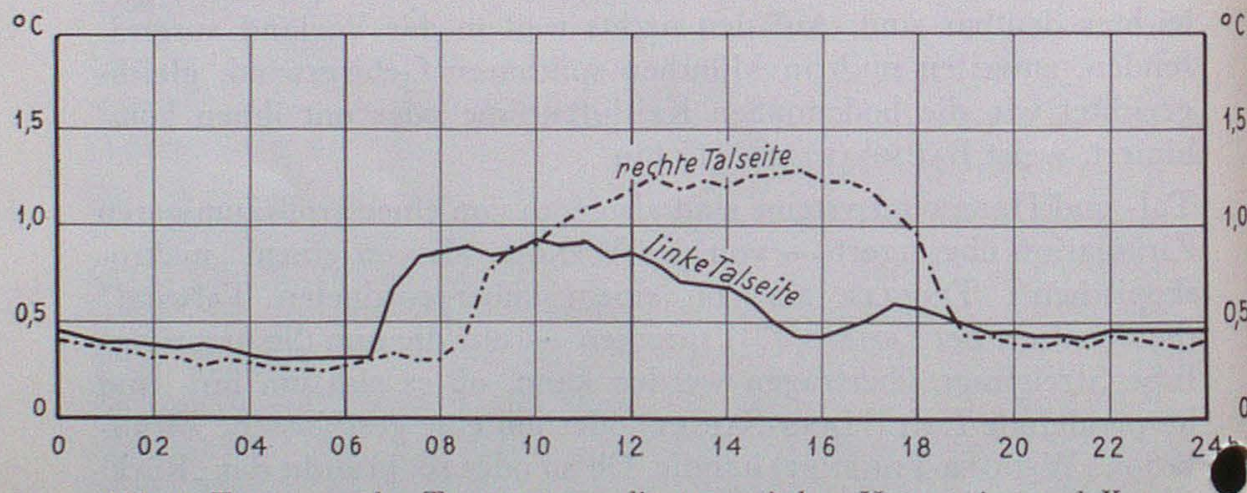

Abb. 8. Tagesgang des Temperaturgradienten zwischen Hangstation und Kammstation jeder Talseite im September (Mittel aus neun Schönwettertagen) aus [28]

diejenige im Talgrund gegenüber, so muß man wohl unterscheiden, ob es sich um Phasen handelt, während denen zwischen Hang- und Talstation ein Luftaustausch besteht, oder solchen, während welcher Hang und Tal nicht mehr vom selben Windsystem bestrichen werden. Die "Temperaturinversion" trägt sicher nichts zum nächtlichen Hangabwind bei. Zu Beginn des Aufwinds ist ein Gradient von 
$0,5^{0} \mathrm{C} / 100 \mathrm{~m}$ (Abb. 9) ungefähr erreicht, wird aber später im wesentlichen nicht mehr überschritten (Westhang). Hingegen wächst bei Nachlassen der Einstrahlung am Osthang, der stärker unter dem Einfluß des Gebirgs- und Talwindes steht, der Gradient auf $1^{\circ} \mathrm{C}$ pro $100 \mathrm{~m}$ und mehr an, wodurch dann die erste Hangabwindkompo-

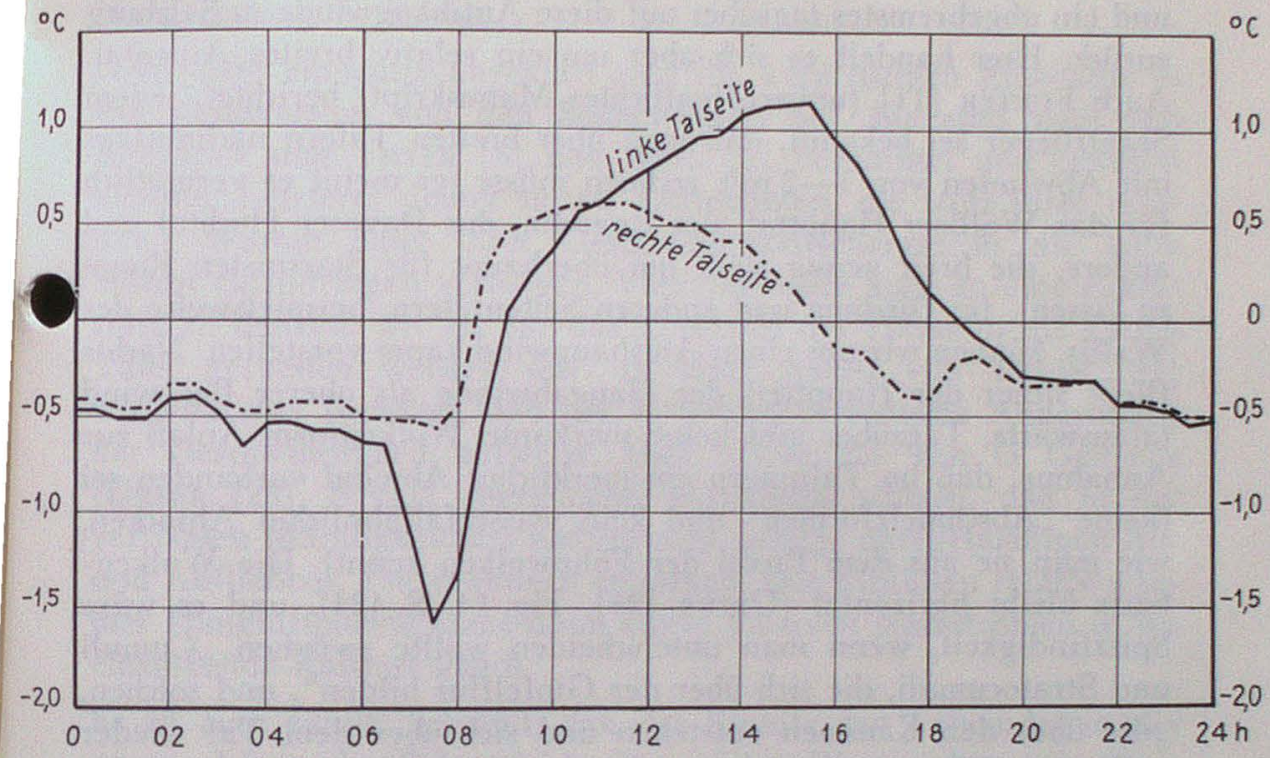

Abb. 9. Tagesgang des Temperaturgradienten zwischen Talstation und Hangstation im September (Mittel aus neun Tagen) aus [28]

nente vor Sonnenuntergang zustande kommt. Auf der gegenüberliegenden Seite fällt das Erreichen der Isothermie ungefähr mit der Winddrehung zusammen.

Vielleicht liegt es an der Lage der fünf Stationen zueinander, daß wir Defants Theorie nicht eindeutig bestätigen können. Wahrscheinlicher scheint allerdings, daß überhaupt nicht nur mit einem einzigen Element, der Lufttemperatur, operiert werden kann. So weiß man z. B. noch kaum etwas darüber, wie sich die Überhitzung des Bodens (Turner [24]) bzw. seine Unterkühlung (Sterten und Knudsen [23]) und die dem Boden aufliegenden Luftschichten auf die lokalen und damit indirekt auf die regionalen Luftversetzungen auswirken. Andererseits beobachtete BERG [2] den Talwind auch über Schneedecke von $0^{0}$ bei Lufttemperaturen von etwa $5^{0}$, dies allerdings in einem bewaldeten Tal mit vom Schnee befreiten Bäumen. 
Offen lassen müssen wir auch die Frage, ob zum Ausgleich der Hangwinde im Talinnern nachts eine aufsteigende und tagsüber eine absinkende Komponente vorhanden sei (WAgner [32]). Für das Dischma bezweifeln wir beides. Buettner und Thyer [3] haben im Carbon River Valley auch nichts derartiges beobachtet. Hingegen führt Eкнавт [9] ein beschleunigtes Steigen der Pilotballons nachts und ein abgebremstes tagsüber auf diese Antihangwinde in Salzburg zurück. Hier handelt es sich aber um ein relativ breites Alpental. Auch Fueter [11] (unveröffentlichtes Manuskript) berichtet, jedem Segelflieger sei bekannt, daß man über breiten Tälern nachmittags mit Abwinden von $1-2 \mathrm{~m} / \mathrm{s}$ rechnen müsse (er meint es vermutlich für das Walliser Haupttal, das Engadin, das Davoser Hochtal und andere, die breit genug sind, um überhaupt für Startpisten Raum zu lassen). Im Dischma und anderen Seitentälern, beispielsweise des Wallis, können wir uns einen Antihangwind kaum vorstellen. Nachts fließt sicher der Hauptteil der Hangabwinde als oberer Bergwind talauswärts. Tagsüber gibt keine markante Wolkenform Anlaß zur Annahme, daß im Talinnern ein merklicher Abwind vorhanden sei (keine "Abschmelzformen" und kein wasserfallähnliches Absacken, wie man sie aus dem Profil der Föhnwolken kennt). Die Wolkenbasis bleibt horizontal (Urfer [28], Fig. 14, S. 424), und es wäre Spitzfindigkeit, wenn man unterscheiden wollte zwischen "Cumuli und Stratocumuli, die sich über der Gipfelflur bilden", und solchen, „die über den Kämmen entstehen und sich über dem Tal wieder auflösen". Schon der schwächste Gradientwind versetzt sie horizontal, ohne daß sie über dem Talinneren gleich in sich zusammenfallen müßten.

Die Querwindzirkulation zur Zeit der Windwechsel am Morgen und am Abend, wie sie auch von Moll [18], Jelinek [14], Berg [2], Ekhart [8], Buettner und Thyer [3], Fueter [11], Van Arsdel [31], MacHattie [16] und anderen beobachtet, von Gleeson [13] in seiner Theorie angenommen worden ist, möchten wir im Dischma nicht als geschlossen bezeichnen. Am Morgen müßte am Thermogramm und an der potentiellen Äquivalenttemperatur der rechtsseitigen Hangstation etwas davon zu beobachten sein, wenn sehr viel wärmere Luft vom Gegenhang herantransportiert würde, und am Abend würde dort der Aufwind nicht schon vor Sonnenuntergang zusammenbrechen, wenn der Querwind einen durchgreifenden Einfluß auszuüben vermöchte. Am Morgen spricht außerdem die auffallend rasche Reaktion des Hangaufwindes auf den Sonnenaufgang (oft nur 5-10 Minuten Anlaufzeit) dafür, daß es sich zunächst nur um kleinräumige Luftversetzungen handelt. 
Daß der nächtliche Bergwind in zwei übereinandergelagerten Strömen talaus fließt, entnehmen wir nicht wie MolL [18] und EKHART $[7,8]$ Pilotballon-Aufstiegen, sondern dem Vergleich der potentiellen Äquivalenttemperaturen. Über beider Mächtigkeit und Lage können wir allerdings nicht mehr aussagen, als daß der untere Kaltluftfluß keine 170 Meter (Lage der untersten Hangstation) am Hang entlang hochsteige.

Noch nicht eindeutig abgeklärt sind die kurzfristigen Einbrüche in die Talinversion von den Seitenhängen her. Sie sind auch von Buettner und Thyer [3] und Berg [2] beobachtet und erwähnt worden. Letzterer deutet sie als Luftquanten, die trotz ihrer relativen Wärme dank ihrer Geschwindigkeitsenergie in die kalte Schicht einzudringen vermögen. Vielleicht wäre auch an eine Erklärung zu denken, wie sie für das Einfallen des Föhns in die kalten Täler gefunden worden war. In einer Arbeit von Tyson [27] wird darüber berichtet, daß der Bergwind periodische Änderungen der Geschwindigkeit aufweise. Dies würde die von G. Gensler (URFER [28], S. 415) abgegebene Vermutung stützen, daß in der vertikalen Mächtigkeit des Kaltluftsees, infolge unregelmäßigen $\mathrm{Zu}$ und Abfließens, Fluktuationen entstehen. Das Einfallen relativ warmer Hangluftpakete käme dann einem passiven Nachströmen in die einsinkende Kaltluft gleich.

Es ist auch bereits bekannt, daß der Einsatz des Talwindes in der ganzen Länge des Tales gleichzeitig erfolgt. Dies möchten wir allerdings nicht vom „gleichmäßigen Ansteigen des Talbodens“ (EкнART [9]) abhängig sehen. Gerade das Dischma weist ein paar sehr markante Stufen auf. Vielmehr spiegelt sich darin die Abhängigkeit der Einsatzzeit des Talwindes vom Sonnenaufgang, der ja auch seinerseits in der ganzen Länge des Tales praktisch zur selben Zeit erfolgt. Daher ist auch die Einsatzzeit der Talwinde in Seitentälern ganz allgemein genauer definiert als in weniger homogenen Haupttälern, wie etwa dem Davoser Hochtal (SchüEPP und URFER [21]) oder dem Inntal. Allerdings wird die Tatsache, daß der Talwind in der ganzen Länge des Tales gleichzeitig einsetzt, von anderen Autoren dahin gedeutet, daß eben in Nebentälern kleinere Luftmassen versetzt werden als in Haupttälern.

Während in den späteren Nachtstunden das Tal- und Hangwindsystem eine Symmetrie besitzt, werden wir diese von Sonnenaufgang bis Stunden nach Sonnenuntergang vergeblich suchen. (Auch bei Buettner und Thyer [3] sowie bei MacHattie [16] kommt diese Asymmetrie gut zum Ausdruck.) Die Besonnungsverhältnisse rufen 
eine solche Vielfalt in den Variationen der Windrichtungen und -geschwindigkeiten hervor, daß hier vom ursprünglichen PrandtlDefantschen System (zitiert in Geiger [12], S. $427 \mathrm{ff}$.) nur noch Bruchstücke übrig bleiben.

\section{Danksagungen}

Den Herren Prof. Dr. A. Kurth, Dr. W. Nägeli und Dr. H. Turner (EAFV) danke ich für die Úberlassung der mesoklimatischen Meßdaten aus dem Dischmatal und für ihre tatkräftige Unterstützung meiner Arbeit, Herrn J. SteinemanN (EAFV) für seine Bemühungen, in einer möglichst klaren graphischen Darstellung allen Variationen des Strömungsschemas gerecht zu werden. Die Schweizerische Meteorologische Zentralanstalt in Zürich stellte im Jahre 1966 aus dem Kredit des Nationalfonds finanzielle Mittel für Versuche mit Frei- und Fesselballons im Dischmatal zur Verfügung, wofür Herrn Direktor R. SchNeider und Herrn Dr. M. Schü̈pp bestens gedankt sei. Herr Dr. R. Widmer hat für diese Untersuchungen von Mitte Juli bis Mitte August 1966 einen Großteil seiner Sommerferien geopfert; infolge anhaltend zyklonaler Wetterlagen konnten typische Verhältnisse leider nur an spärlichen wolkenlosen Morgen- und Abendstunden studiert werden, jedoch gelangen während einer Schönwetterperiode im September 1966 Herrn Dr. M. Schüвpp und der schwedischen Meteorologin E. ZACKE mittels beinahe ausgewichteter Pilotballone einige wertvolle ergänzende Messungen und Beobachtungen.

\section{Literatur}

1. Baumgartner, A.: Wald im Alpenvorland aus klimatologischer Sicht. Allg. Forstz. 22, 595-598 (1967).

2. Berg, H.: Beobachtungen des Berg- und Talwindes in den Allgäuer Alpen. Ber. Dtsch. Wetterd. US-Zone 6, Nr. 38, 105 (1952).

3. Buettaer, K., and N. Thyer: On Valley and Montain Winds. Final report AF contract 19(604) 2289, Dept, of Meteor, and Climat., Univ, of Washington, Seattle, 28 pp. (1959).

4. Buettmer, K., and N. Thyer: Valley Winds in the Mt. Rainier Area. Dept. of Atmosph. Sciences, Univ. of Washington, Seattle (1965) bzw. Arch. Met. Geoph. Biokl., B, 14, 125-147 (1966).

5. BURger, A., und E. EkHART: Über die tägliche Zirkulation der Atmosphäre im Bereiche der Alpen. Gerl. Beitr. Geoph. 49, 341 (1937).

6. Defant, F.: Zur Theorie der Hangwinde, nebst Bemerkungen zur Theorie der Berg- und Talwinde. Arch. Met. Geoph. Biokl., A, 1, 421 (1949).

7. Ekhart, E.: Nachtpiloten. Beitr. Physik fr. Atm. 18 (1931).

8. Eкhart, E.: Die periodischen Tageswinde in einem Quertal der Alpen. Beitr. Physik fr. Atm. 21 (1934).

9. EkHart, E.: Strömungsaerologie der Talwinde. Met. Z. 61, H. 7 (1944).

10. ЕкнаRт, E.: Úber den täglichen Gang des Windes im Gebirge. Arch. Met. Geoph. Biokl., B, 4, 431 (1952/53). 
11. Fueter, P.: Die tagesperiodischen Winde in Gebirgstälern. Unveröff. Manuskript, Armeewetterdienst, 23 S., 1942.

12. Geiger, R.: Das Klima der bodennahen Luftschicht, 4. Aufl. Braunschweig: Friedr. Vieweg \& Sohn, 1961.

13. Gleeson, T. A.: On the Theory of Cross-Valley Winds Arising from Differential Heating of the Slopes. J. Meteorol. 8, 398-405 (1951).

14. Jelinek, A.: Untersuchungen periodischer Tageswinde in Südtirol. Beitr. Physik fr. Atm. 21, 223 (1934).

15. KuHN, W.: Horizontale Luftdruckgradienten in den Alpen nach Messungen im Gebiet des Kistenpasses. Verh. Schweiz. Nat'forsch. Ges., Altdorf, 1954, $105-109$ (1954).

16. MacHattie, L. B.: Kananaskis Valley Winds in Summer. J. Appl. Meteorol. 17, No. 3 (1968).

17. MäDER, F.: Untersuchung über die Windverhältnisse in Bodennähe bei verschiedenen Wetterlagen. Veröff. Schweiz. Meteorol. Zentralanst., Nr. 9, 42 S. (1968).

18. Moll, E.: Aeorologische Untersuchungen periodischer Gebirgswinde in V-förmigen Alpentälern. Beitr. Physik fr. Atm. 22 (1935).

19. NäGELI, W.: Über die Windverhältnisse im Bereich gestaffelter Windschutzstreifen. Mitt. Schweiz. Anst. forst. Versuchswesen 41, H. 5, 219-300 (1965).

20. SchüEPp, W.: Beobachtungen und Überlegungen zum tagesperiodischen Luftmassenaustausch. Arch. Met. Geoph. Biokl., A, 5, 36-43 (1952).

21. Schüepp, M., und Ch. Urfer: Die Windverhältnisse im Davoser Hochtal. Arch. Met. Geoph. Biokl., B, 12, 337-349 (1963).

22. Sterten, A. K.: Alte und neue Berg- und Talwindstudien. 8. Int. Tagung Alpine Meteorol. Villach 1964, Carinthia II, 24. Sonderheft, 186-194 (1965).

23. Sterten, A. K., and J. Knudsen: Local and Synoptic Meteorological Investigations of the Mountain and Valley Wind System. Forsvarets Forskningsinstitutt, Norwegian Defence Research Establishment, Kjeller-Lillestrøm, Intern Rapport K-242, 1961, 139 pp.

24. Turner, H.: Maximaltemperaturen oberflächennaher Bodenschichten. Wetter u. Leben 10, 1-12 (1958).

25. Turner, H.: Die globale Hangbestrahlung als Standortsfaktor bei Aufforstungen in der subalpinen Stufe. Mitt. Schweiz. Anst. forstl. Versuchswesen 42, H. 3, 109-168 (1966).

26. Turner, H.: Mikroklimate und ihre Beziehungen zu Geländeformung und Vegetation in einem Schweizer Hochgebirgstal. Vortr. Meteorol. Koll. Univ. München, Dezember 1967 (unveröff. Manuskript).

27. Tyson, P. D.: Velocity Fluctuations in the Mountain Wind. J. Atmosph. Sci. 25, 381-384 (1968).

28. Urfer-Henneberger, Ch.: Wind- und Temperaturverhältnisse an ungestörten Schönwettertagen im Dischmatal bei Davos. Mitt. Schweiz. Anst, forstl. Versuchsw. 40, H. 6, 389-441 (1964).

29. Urfer-Henneberger, Ch.: Nachweis der lokalen Strömungsverhältnisse im Dischmatal mit Hilfe der potentiellen Äquivalenttemperatur. Verh. Schweiz. Nat'forsch. Ges., Zürich, 1964, 99-100 (1964). 
30. Urfer-Henneberger, Ch.: Zeitliche Gesetzmäßigkeiten des Berg- und Talwindes. 9. Int. Tagung Alpine Meteorol. Brig 1966, Veröff. Schweiz. Meteorol. Zentralanst., Nr. 4, 245-252 (1967).

31. van Arsdel, E. P.: The Nocturnal Diffusion and Transport of Spores. Phytopathology 57, No. 11, 1221-1229 (1967).

32. Wagner, A.: Neue Theoric des Berg- und Talwindes. Met. Z. 49, 9 (1932).

33. Wagner, A.: Theorie und Beobachtung der periodischen Gebirgswinde. Gerl. Beitr. Geoph. 52, 408-449 (1938).

Anschrift der Verfasserin: Dr. Charlotte Urfer-Henneberger, Sonnhaldenstraße 19, CH-8302 Kloten ZH. 